\title{
Dosimetric Comparison of Superflab and Specially Prepared Bolus Materials Used in Radiotherapy Practice
}

\author{
Serhat Aras ${ }^{1}$ (D), İhsan Oğuz Tanzer² (1), Türkan İkizceli³ (1) \\ ${ }^{1}$ Medical Imaging Programme, University of Health Sciences, İstanbul, Turkey \\ ${ }^{2}$ Biomedical Technology Programme, University of Health Sciences, İstanbul, Turkey \\ ${ }^{3}$ Department of Radiology, University of Health Sciences, Haseki Training and Research Hospital, İstanbul, Turkey
}

\begin{abstract}
Objective: This study compares standard commercial bolus material (Superflab) to custom prepared silicone dental impression material (CDIM) and play dough material (PDM) with respect to dosimetric properties and applicability by using ion chamber measurement and calculated dose values.

Materials and Methods: The CDIM bolus was prepared by mixing dental impression silicone material with enough water to maintain a density of about $1.0 \mathrm{~g} / \mathrm{cm}^{3}$. The prepared bolus material is applied on an RW3 solid phantom by covering $10 \times 10 \mathrm{~cm}^{2}$ area with $0.5-1 \mathrm{~cm}$ thickness. Ion chamber measurements were performed separately with and without bolus material application. The setup was scanned in CT and the same procedure was repeated in the TPS using the scan data, in which the Pencil Beam Convolution dose calculation algorithm was used. To compare the effect of bolus material on tissue, the Superflab bolus and CDIM bolus were applied with $1 \mathrm{~cm}$ of thickness on postmastectomy scar and dose calculations on TPS were performed. Results: After comparison of the dosimetric values for Superflab, CDIM and PDM, we obtained statistically meaningful results between superflab and CDIM. For PDM, the results obtained with TPS and ion chamber measurements indicated that, it is not suitable to use in radiotherapy application due to its material properties. For the simulated skin dose values obtained at five random points on the scar tissue, the comparison of Superflab and CDIM TPS calculation results were not statistically significant.

Conclusion: The CDIM is easy to prepare and apply on irregular mastectomy scar tissue and it prevents formation of air gaps in the application surface. Especially for curved anatomical regions such as scar tissue, inclusion of the bolus material in treatment planning protocol will reduce dose uncertainty in application. It is safe to use CDIM as an alternative to Superflab in radiotherapy application, whereas PDM is not useful in clinical practice due to its material properties.
\end{abstract}

Keywords: Bolus material, superflab, dental impression material, play dough, radiotherapy

Cite this articles as: Aras S, Tanzer İO, İkizceli T. Dosimetric Comparison of Superflab and Specially Prepared Bolus Materials Used in Radiotherapy Practice. Eur J Breast Health 2020; 16(3): 167-170.

\section{Introduction}

The Bolus Material is defined as a tissue equivalent material that maximizes, reduces or adds radiation dose in an irradiated area according to the ICRU Report 24 (1). Bolus is used in radiotherapy (RT) to increase skin dose for photon beams (2-4).

Bolus materials are used in high-energy RT in order to overcome the skin-sparing effect of high energy radiation beams, which prevents delivery of sufficient dose to the skin. Several types of commercially available bolus materials are often used in RT units (5). It is important in clinical practice that the bolus material is sufficiently elastic and deformable in order to conform to the surface and not adversely affected by high dose levels, be durable, nontoxic, and cost effective (6). Bolus materials should be nearly tissue-equivalent and allow sufficient surface dose boost. Superflab is a commercial bolus material widely accepted in RT clinics worldwide. It is made of a proprietary synthetic gel, resulting in a mouldable material that does not suffer inelastic strain from normal stresses. Consequently, Superflab does not have to be bagged or wrapped in plastic film to maintain its shape during treatment. To optimally support dose build-up for varying surface contours and target volumes, several sizes are available. The material density of Superflab is $1.02 \mathrm{~g} / \mathrm{cm}^{3}$ which is similar to water in approximating tissue-equivalence and well accepted clinically (7). In practice though, Superflab is not effective in making sufficient contact with irregular anatomical surface on the patient's skin. This is the case particularly around the nose, ear, and scalp, resulting in 
air gaps, thus creating the skin sparing effect which reduces both the maximum and surface dose (8). In literature, various bolus materials are used, such as; thermoplastic sheets, blue water phantoms, 3D customized bolus $(9,10)$.

The purpose of this study is to compare the ion chamber measurements and calculated dose values of a standard commercial bolus material (Superflab) with two other materials which are-custom prepared silicone dental impression material (CDIM) and play dough material (PDM). In addition, we also compare the treatment simulation of skin dose values between Superflab and CDIM on surgical scar using twenty 20 randomly selected and anonymous breast cancer patients who underwent modified radical mastectomy, using the treatment planning system (TPS).

\section{Materials and Methods}

\section{Preparation of bolus materials}

Superflab (Radiation Products Design Inc, Albertville, MN) bolus is widely used in clinical practice and commercially available. Since commercially available silicone dental impression material is not flexible enough for satisfactory application right out of the box, we prepared a CDIM; $900 \mathrm{~mL}$ of silicone dental impression material (Detax Exaplast) with a density of $0.95-1.02 \mathrm{~g} / \mathrm{cm}^{3}$ was mixed with water in such a way that the material density of the CDIM was close to $1.0 \mathrm{~g} / \mathrm{cm}^{3}$ and the resulting material was sufficiently flexible for application. The amount of water needed for the desired flexibility of the CDIM was determined to be $100 \mathrm{~mL}$ after several trial samples were chemically analysed.

The play dough material (PDM) used in this study is mainly a solid-liquid mixture and commercially available. PDM contains water, starch-based binder, salt, oil, preservative, hardener, moisturizer, perfume and food colouring and has a density around $1.0 \mathrm{~g} / \mathrm{cm}^{3}$.

\section{Data acquisition on solid phantom}

Bolus materials covering an area of $10 \times 10 \mathrm{~cm}^{2}$ with $0.5 \mathrm{~cm}$ and $1 \mathrm{~cm}$ thickness were prepared and applied on RW3 solid phantom for the ion chamber measurements. Using the linear accelerator (Siemens Primus, Germany) in our clinic, $6 \mathrm{MV}$ photon energy is applied to give 100 monitor unit (MU) in order to measure the dose values at a depth of $5 \mathrm{~cm}$ of the solid phantom. Measurements were performed separately with and without bolus material application. Next, a computed tomography (CT) (Siemens Somatom Definition, Germany) scan with $3 \mathrm{~mm}$ slice thickness is performed on the measurement setting. Using the CT scan data, same procedure was repeated in the TPS (Eclipse $\mathrm{V}_{8.908}$ Varian, USA) in which the Pencil Beam Convolution (PBC) dose calculation algorithm was used. The absorbed dose values at 5 $\mathrm{cm}$ depth of the solid phantom were calculated (1 Gy/fr and $6 \mathrm{MV}$ ).

\section{Obtaining calculated data on skin}

For the skin dose simulation on surgical scars, CT images for a group of 20 randomly selected anonymous breast cancer patients who underwent modified radical mastectomy operation in our clinic were selected for evaluation. Ethics committee approval was not required since this was not a clinical study performed on patients, but a dosimetric simulation study. Informed Consent was not required since the dosimetric simulation study was performed on anonymous patient data. In the TPS, Superflab bolus and CDIM bolus are applied with $1 \mathrm{~cm}$ of thickness on each of the post-mastectomy scars in order to increase the dose on the scar. This thickness was preferred because the $6 \mathrm{MV}$
$1.5 \mathrm{~cm}$. The effect of the bolus materials on the TPS dose calculation values are compared. PDM bolus was not used for the breast cancer patient simulation due to its dosimetric and material properties which were not suitable for practical clinical application.

\section{Simulated radiotherapy procedure using different bolus materials}

In the TPS, two different treatment plans were prepared for the cases where Superflab and CDIM is used. Parameters for the treatment simulation are Pencil Beam Convolution dose calculation algorithm, $6 \mathrm{MV}$ photon energy, skin source distance of $100 \mathrm{~cm}$, scar $+0.5 \mathrm{~cm}$ multi leaf collimator (MLC) margin and 2Gy dose, and $5 \mathrm{~mm}$ slice thickness in CT images. For the CT image set, absorbed dose at five randomly chosen points on the surgical scars were calculated in TPS. Since a virtual bolus [0-400 Hounsfield Unit (HU)] is used and dose calculations are performed on TPS, an ethics committee approval was not needed.

\section{Statistical analysis}

The treatment simulation data with bolus materials for the 20 patient CT image set was subject to statistical analysis with Statistical Package for Social Sciences Software version 18 (IBM SPSS Corp.; Armonk, NY, USA) using Student's t-test. The calculated $\mathrm{p}<0.05$ and considered statistically significant.

\section{Results}

Both for the measurements made with the ion chamber system and for the results obtained in the TPS, with and without bolus material, the percent dose differences between the absorbed dose values (Gy) were calculated. Each measurement was repeated 3 times and the average was taken. For $0.5 \mathrm{~cm}$ thick Superflab bolus, the absorbed dose reduction was calculated as $1.28 \%$ in the ion chamber measurements and $2.41 \%$ in the TPS; for $1,0 \mathrm{~cm}$ thick bolus, the absorbed dose reduction was calculated as $2.80 \%$ in the ion chamber measurements and $5.80 \%$ in the TPS. For CDIM bolus of $0.5 \mathrm{~cm}$ the absorbed dose reduction was calculated as $3.15 \%$ in the ion chamber measurements and $4.24 \%$ in the TPS; for $1,0 \mathrm{~cm}$ thick bolus, the absorbed dose reduction was calculated as $3.42 \%$ in the ion chamber measurements and $3.88 \%$ in the TPS. Finally, for the play dough bolus of $0.5 \mathrm{~cm}$ the absorbed dose reduction was calculated as $2.04 \%$ in the ion chamber measurements and $3.21 \%$ in the TPS; for $1.0 \mathrm{~cm}$ thick bolus, the absorbed dose reduction was calculated as $5.13 \%$ in the ion chamber measurements and $6.88 \%$ in the TPS (Table 1 ).

For different bolus materials and simulated dose values calculated at 5 random points within 20 mastectomy patient CT dataset, it was observed that the difference in absorbed dose value between the Superflab and CDIM bolus had less than $1 \%$ difference in the TPS. The differences in dose values were not statistically significant (Table 2).

\section{Discussion and Conclusion}

In our study we have compared the dosimetric values for Superflab, CDIM and PDM. Commercially available dental impression material is inflexible out of the box to be of suitable use in the clinic. We created a special mixture with readily available dental impression material and water, creating a flexible material suitable for application as bolus and obtained statistically meaningful results $(\mathrm{p}<0.05)$ when compared with Superflab. In the literature, it was reported that resulting radiation doses were similar when Play-Dough and Superflab bolus were used and doses resulting from Play-Dough bolus approximated those of Superflab (11). However, in our study, when the PDM is applied 
Table 1. Absorbed dose values and percentage differences, measured at a depth of $5 \mathrm{~cm}$, when using Superflab and CDIM for bolus material thickness of $0.5 \mathrm{~cm}$ and $1.0 \mathrm{~cm}$

\begin{tabular}{|c|c|c|c|c|c|c|c|c|}
\hline & \multicolumn{4}{|c|}{ Absorbed Dose } & \multicolumn{4}{|c|}{ Percent Difference (\%) } \\
\hline & \multicolumn{2}{|c|}{$\begin{array}{l}\text { Ion Chamber } \\
\text { Measured Dose (Gy) }\end{array}$} & \multicolumn{2}{|c|}{$\begin{array}{c}\text { TPS } \\
\text { Calculated Dose (Gy) }\end{array}$} & \multicolumn{2}{|c|}{ Ion Chamber } & \multicolumn{2}{|c|}{ TPS } \\
\hline & $0.5 \mathrm{~cm}$ & $1 \mathrm{~cm}$ & $0.5 \mathrm{~cm}$ & $1 \mathrm{~cm}$ & $0.5 \mathrm{~cm}$ & $1 \mathrm{~cm}$ & $0.5 \mathrm{~cm}$ & $1 \mathrm{~cm}$ \\
\hline No bolus & 0.8756 & 0.8756 & 0.8750 & 0.8750 & & & & \\
\hline Superflab & 0.8644 & 0.8510 & 0.8510 & 0.8240 & 1.28 & 2.80 & 2.41 & 5.80 \\
\hline CDIM & 0.8480 & 0.8476 & 0.8450 & 0.8410 & 3.15 & 3.42 & 4.24 & 3.88 \\
\hline PDM & 0.8577 & 0.8307 & 0.8440 & 0.8050 & 2.04 & 5.13 & 3.21 & 6.88 \\
\hline
\end{tabular}

\section{Table 2. Comparison of calculated TPS dose values for Superflab and CDIM at five random point locations on post-mastectomy scar area}

\begin{tabular}{|llcc|}
\hline Location & Material & Mean dose $(G \mathbf{y}) \pm \mathbf{s d}$ & P \\
\hline Point 1 & Superflab & $1.0159 \pm 2.22$ & 0.271 \\
& CDIM & $1.0150 \pm 2.27$ & \\
Point 2 & Superflab & $1.0244 \pm 2.50$ & 0.481 \\
& CDIM & $1.0235 \pm 2.49$ & \\
Point 3 & Superflab & $1.0254 \pm 2.62$ & 0.669 \\
& CDIM & $1.0262 \pm 2.73$ & \\
Point 4 & Superflab & $1.0254 \pm 2.73$ & 0.754 \\
& CDIM & $1.0260 \pm 2.77$ & \\
Point 5 & Superflab & $1.0182 \pm 3.12$ & 0.476 \\
& CDIM & $1.0163 \pm 3.38$ & \\
\hline
\end{tabular}

Gy: Gray; CDIM: silicone dental impression material

and analysed with both TPS and ion chamber measurements, the results indicated that it is not appropriate to use PDM in RT application consequently it was not used clinically on our patients.

Radiotherapy related parameters such as dose application technique, area size, beam angle, and the algorithms in use to calculate the dose values between calculated and applied dose, cause, either a linear or non-linear dose increase in skin tissue between the bolus application sessions. Therefore, further dosimetric studies are required in order to assess the accuracy of a certain beam energy, bolus thickness and the algorithm used in the TPS technique for dose calculation. Although there are various suggestions in the literature for RT planning techniques, there are very few comments and suggestions regarding bolus use. The optimal thickness of the bolus material and appropriate remains uncertain and vary from one RT centre to another (12).

As noted in the literature, the dose taken by the skin is lower than the dose defined for the target due to difference in RT plans' usage of high-energy beams. Bolus materials are widely used to bring the absorbed skin dose to the desired level. According to the desired clinical outcome, dose absorption of the skin can be determined, and the bolus used in respective treatment fractions can be decided upon (13). During RT it is important to accurately detect chest wall and skin surface depth in order to limit early and late skin reaction and to prevent cancerous recurrence close to the skin surface. The dose contribution of bolus material to skin and subcutaneous tissue is especially important $(14,15)$.

For breast cancer patients, RT is an essential part of the treatment protocol. During post-mastectomy RT, tissue-equivalent bolus materials with enough thickness are often used in order to provide a buildup dose on the skin and chest wall. In our simulation study for the patients who had undergone mastectomy and RT, for the five randomly chosen points on the scar tissue the comparison of Superflab and CDIM TPS calculation results were not statistically significant ( $>0.05$ ). In the phantom study we have seen that both materials have similar properties. We observed that for the RT treatment of breast cancer patients who underwent modified radical breast mastectomy, the CDIM had the closest effect on the absorbed dose compared to the Superflab bolus.

It is important to evaluate the difference between calculated and measured skin dose and to compare patient plans. Parameters such as irradiation technique, area, beam angle, presence of air pockets and the use of bolus material affect the amount of skin dose $(16,17)$. Optimizing the use different bolus materials is also clinically useful. In the calculation of skin dose, while treatment planning systems do not fully account for all factors contributing to surface dose, new techniques such as Monte Carlo and 3D modelling algorithms are able to calculate the skin dose with higher accuracy $(18,19)$.

In clinical practice, bolus thickness required to increase the surface dose is optimized according to the skin type and build-up zone dosimetry $(20,21)$. The actual thickness of the bolus material for a patient is decided by the by radiation oncologist and medical physicist during dose planning in the Treatment Planning System (TPS). If a high amount of dose distribution is requested on the skin or near the skin, a bolus thickness of $1 \mathrm{~cm}$ is preferred. On the other hand, in certain cases, $0.5 \mathrm{~cm}$ bolus thickness is preferred in order to decrease radiation related complications

The CDIM is easy to prepare and apply on irregular mastectomy scar tissue and it prevents formation of air gaps in the application surface. Especially for curved anatomical regions such as scar tissue, inclusion of the bolus material in treatment planning protocol will reduce dose 
uncertainty in application. PDM is not useful in clinical practice for two reasons, the dosimetry results for PDM shows that it absorbed more dose than required compared to Superflab bolus. Second, PDM is challenging to use routinely with daily fraction treatments as bolus material, as it hardens when in contact with air and this might create undesired air gaps in uneven skin applications. It is safe to use CDIM as an alternative to Superflab in radiotherapy application, whereas PDM is not useful in clinical practice.

Ethics Committee Approval: Ethics committee approval was not required since this was not a clinical study performed on patients, but a dosimetric simulation study.

Informed Consent: Informed Consent was not required since the dosimetric simulation study was performed on anonymous patient data.

Peer-review: Externally peer-reviewed.

Author Contributions: Concept - S.A., I.O.T., T.I.; Design - S.A., I.O.T.; Supervision - S.A., I.O.T., T.I.; Funding - S.A.; Materials - S.A.; Data Collection and/ or Processing - S.A.; Analysis and/or Interpretation - S.A., I.O.T., T.I.; Literature Review - S.A., I.O.T., T.I.; Writer - S.A., I.O.T., T.I.; Critical Review - S.A., I.O.T., T.I.

Acknowledgements: The authors would like to thank Dr. Özge Nurcan Tanzer for helpful comments.

Conflict of Interest: The authors have no conflicts of interest to declare.

Financial Disclosure: The authors declared that this study has received no financial support.

\section{References}

1. Journal of the International Commission on Radiation Units and Measurements. Report 1976; 24: 13. [CrossRef]

2. Babic S, Kerr A, Westerlan M, Gooding J, Schreiner J. Examination of Jeltrate Plus as a tissue equivalent bolus material. J Appl Clin Med Phys 2002; 3: 170-175. (PMID: 12132938) [CrossRef]

3. Vyas V, Palmer L, Mudge R, Jiang R, Fleck A, Schaly B, Osei E, et al. On bolus for megavoltage photon and electron radiation therapy. Med Dosim 2013; 38: 268-273. (PMID: 23582702) [CrossRef]

4. Banaee N, Nedaie H.A, Nosrati H, Nabavi M, Naderi M. Dose measurement of different bolus materials on surface dose. Journal of Radioprotection Research 2013; 1: 10-13. [CrossRef]

5. Khan FM. The physics of radiation therapy. 4th ed. Philadelphia, Lippincott Williams \& Wilkins, 2010.

6. Kong M, Holloway L. An investigation of central axis depth dose distribution perturbation due to an air gap between patient and bolus for electron beams. Australas Phys Eng Sci Med 2007; 30: 111-119. (PMID: 17682400) [CrossRef]
7. Solanki A, M A, A H, Kumar HS. Reduction of Dose to the Contralateral Breast by Superflab Use in Radiation Therapy for Mammary Carcinomas. Asian Pac J Cancer Prev 2017; 18: 1025-1029.

8. Khan Y, Villarreal-Barajas JE, Udowicz M, Sinha R, Muhammad W, Ahmed N. Abbasi et al. Clinical and dosimetric implications of air gaps between bolus and skin surface during radiation therapy. J Cancer Ther 2013; 4: 1251-1255. [CrossRef]

9. Visscher S, Barnett E. Comparison of bolus materials to highly absorbent polypropylene and rayon cloth. J Med Imaging Radiat Sci 2017; 48: 5560. (PMID: 31047211) [CrossRef]

10. 1990 Recommendations of the International Commission on Radiological Protection. ICRP Publication 60. Ann. ICRP 1991; 21: 4-11. [CrossRef]

11. Walker M, Cohen N, Menchaca D. Play-Doh and water-soaked gauze sponges as alternative bolus material for cobalt-60 teletherapy. Vet Radiol Ultrasound 2005; 46: 179-181. (PMID: 15869164) [CrossRef]

12. Yokoyama S, Roberson P, Litzenberg D, Moran J, Fraas B. Surface build up dose dependence on photon field delivery technique for IMRT. J Appl Clin Med Phys 2004; 5: 71-81. (PMID: 15738914) [CrossRef]

13. Andıç F, Ors Y, Davutoglu R, Çifci B, Ispir EB, Erturk E. Evaluation of skin dose associated with different frequencies of bolus applications in post-mastectomy three-dimensional conformal radiotherapy. J Exp Clin Cancer Res 2009; 28: 41. (PMID: 19317895) [CrossRef]

14. Hsu SH, Roberson PL, Chen Y, Marsh RB, Pierce LJ, Moran JM. Assesment of skin dose for breast chest wall radiotherapy as a function of bolus material. Phys Med Biol 2008; 53: 2593-2606. (PMID: 18441412) [CrossRef]

15. Mahdavi H, Jabbari K, Roayaei M. Evaluation of various boluses in dose distribution for electron therapy of the chest wall with an inward defect. J Med Phys 2016; 41: 38-44. (PMID: 27051169) [CrossRef]

16. Hennequin C, Barillot I, Azria D, Belkacémi Y, Bollet M, Chauvet B, et al. Radiotherapy of breast cancer. Cancer Radiother 2016; 20: 139-146. (PMID: 27522187) [CrossRef]

17. Fischbach M, Halg RA, Hartmann M, Besserer J, Gruber G, Schneider U. Measurement of skin and target dose in post-mastectomy radiotherapy using 4 and 6 MV photon beams. Radiat Oncol 2013; 8: 270. (PMID: 24238366) [CrossRef]

18. Sharma SC, Johnson MW. Surface dose perturbation due to air gap between patient and bolus for electron beams. Med Phys 1993; 20: $377-$ 378. (PMID: 8497226) [CrossRef]

19. Butson MJ, Cheung T, Yu P, Metcalfe P. Effects on skin dose from unwanted air gaps under bolus in photon beam radiotherapy. Radiat Meas 2000; 32: 201-204. [CrossRef]

20. Malaescu I, Marin C.N, Spunei M. Comparative study on the surface dose of some bolus materials. Int J Med Phys Clin Eng Radiat Oncol 2015; 4: 348-352. [CrossRef]

21. Court LE, Tishler R, Xiang H, Allen AM, Makrigiorgos M, Chin L. Experimental evaluation of the accuracy of skin dose calculation for a commercial treatment planning system. J Appl Clin Med Phys 2008; 9: 29-35. (PMID: 18449168) [CrossRef] 